\title{
3D Through-Wall Imaging with Unmanned Aerial Vehicles Using WiFi
}

\author{
Chitra R. Karanam \\ University of California Santa Barbara \\ Santa Barbara, California 93106 \\ ckaranam@ece.ucsb.edu
}

\author{
Yasamin Mostofi \\ University of California Santa Barbara \\ Santa Barbara, California 93106 \\ ymostofi@ece.ucsb.edu
}

\begin{abstract}
In this paper, we are interested in the 3D through-wall imaging of a completely unknown area, using WiFi RSSI and Unmanned Aerial Vehicles (UAVs) that move outside of the area of interest to collect WiFi measurements. It is challenging to estimate a volume represented by an extremely high number of voxels with a small number of measurements. Yet many applications are time-critical and/or limited on resources, precluding extensive measurement collection. In this paper, we then propose an approach based on Markov random field modeling, loopy belief propagation, and sparse signal processing for 3D imaging based on wireless power measurements. Furthermore, we show how to design efficient aerial routes that are informative for 3D imaging. Finally, we design and implement a complete experimental testbed and show high-quality 3D robotic through-wall imaging of unknown areas with less than $4 \%$ of measurements.
\end{abstract}

\section{CCS CONCEPTS}

•Computer systems organization $\rightarrow$ Robotics; •Hardware $\rightarrow$ Sensor devices and platforms; $\bullet$ Networks $\rightarrow$ Wireless access points, base stations and infrastructure;

\section{KEYWORDS}

Through-Wall Imaging, 3D Imaging, WiFi, Unmanned Aerial Vehicles, RF Sensing

ACM Reference format:

Chitra R. Karanam and Yasamin Mostofi. 2017. 3D Through-Wall Imaging with Unmanned Aerial Vehicles Using WiFi. In Proceedings of The 16th ACM/IEEE International Conference on Information Processing in Sensor Networks, Pittsburgh, PA USA, April 2017 (IPSN 2017), 12 pages.

DOI: http://dx.doi.org/10.1145/3055031.3055084

\section{INTRODUCTION}

Sensing with Radio Frequency (RF) signals has been a topic of interest to the research community for many years. More recently, sensing with everyday RF signals, such as WiFi, has become of particular interest for applications such as imaging, localization, tracking, occupancy estimation, and gesture recognition [2, 12, 13, 18, 33, 38].

Permission to make digital or hard copies of all or part of this work for personal or classroom use is granted without fee provided that copies are not made or distributed for profit or commercial advantage and that copies bear this notice and the full citation on the first page. Copyrights for components of this work owned by others than ACM must be honored. Abstracting with credit is permitted. To copy otherwise, or republish, to post on servers or to redistribute to lists, requires prior specific permission and/or a fee. Request permissions from permissions@acm.org.

IPSN 2017, Pittsburgh, PA USA

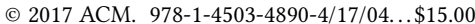

DOI: http://dx.doi.org/10.1145/3055031.3055084
Among these, through-wall imaging has been of particular interest due to its benefits for scenarios like disaster management, surveillance, and search and rescue, where assessing the situation prior to entering an area can be very crucial. However, the general problem of through-wall imaging using RF signals is a very challenging problem, and has hence been a topic of research in a number of communities such as electromagnetics, signal processing, and networking $[11,12,38]$.

For instance, in the electromagnetics literature, inverse scattering problems have long been explored in the context of imaging $[10,14,29]$. Ultra wideband signals have also been heavily utilized for the purpose of through-wall imaging $[3,4,11,36]$. Phase information has also been used for beam forming, time-reversal based imaging, or in the context of synthetic aperture radar [2, 11, 41]. However, most past work rely on utilizing a large bandwidth, phase information, or motion of the target for imaging. Validation in a simulation environment is also common due to the difficulty of hardware setup for through-wall imaging. In [12,31], the authors use WiFi RSSI measurements to image through walls in 2D. They show that by utilizing unmanned ground vehicles and proper path planning, 2D imaging with only WiFi RSSI is possible. This has created new possibilities for utilizing unmanned vehicles for RF sensing, which allows for optimizing the location of the transmitter/receiver antennas in an autonomous way. However, 3D throughwall imaging with only WiFi RSSI measurements, which becomes considerably more challenging than the corresponding 2D problem, has not been explored, which is the main motivation for this paper. It is noteworthy that directly applying the $2 \mathrm{D}$ imaging framework of $[12,31]$ to the $3 \mathrm{D}$ case can result in a poor performance (as we see later in the paper), mainly because the $3 \mathrm{D}$ problem is considerably more under-determined. This necessitates a novel and holistic 3D imaging framework that addresses the new challenges, as we propose in this paper.

In this paper, we are interested in the 3D through-wall imaging of a completely unknown area using Unmanned Aerial Vehicles (UAVs) and WiFi RSSI measurements. More specifically, we consider the scenario where two UAVs move outside of an unknown area, and collect wireless received power measurements to reconstruct a 3D image of the unknown area, an example of which is shown in Fig. 1. We then show how to solve this problem using Markov random field (MRF) modeling, loopy belief propagation, sparse signal processing, and proper 3D robotic path planning. We further develop an extensive experimental testbed and validate the proposed framework. More specifically, the main contributions of this paper are as follows:

(1) We propose a framework for 3D through-wall imaging of unknown areas based on MRF modeling and loopy belief 

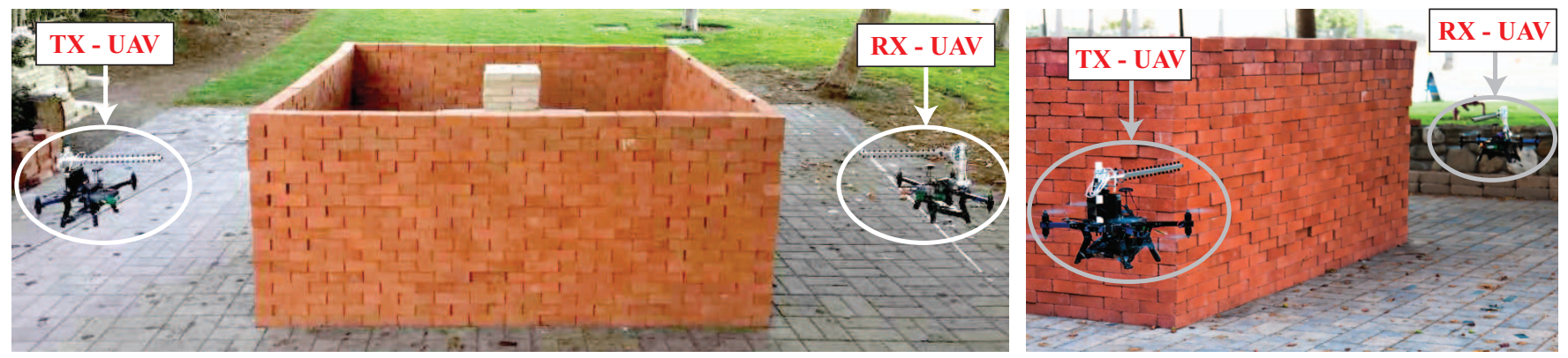

Figure 1: Two examples of our considered scenario where two UAVs fly outside an unknown area to collect WiFi RSSI measurements for the purpose of 3D through-wall imaging.

propagation. In the vision literature, MRF modeling has been utilized in order to incorporate the spatial dependencies among the pixels of an image [22,34]. Furthermore, various methods based on loopy belief propagation [16, 34], iterative conditional modes [22], and graph cuts [27] have been proposed for image denoising, segmentation, and texture labeling. In this paper, we borrow from such literature to solve our 3D through-wall imaging problem, based on sparse signal processing, MRF modeling and loopy belief propagation.

(2) We show how to design efficient robotic paths in 3D for our through-wall imaging problem.

(3) We design and implement a complete experimental testbed that enables two octo-copters to properly localize, navigate, and collect wireless measurements. We then present 3D through-wall imaging of unknown areas using our testbed. Our results confirm that high-quality through-wall imaging of challenging areas, such as behind thick brick walls, is possible with only WiFi RSSI measurements and UAVs. To the best of our knowledge, our 3D imaging results showcase high-quality imaging of more complex areas than what has been reported in the literature with even phase and/or UWB signals.

The rest of this paper is organized as follows. In Section 2, we formulate our 3D through-wall imaging problem and summarize the measurement model. In Section 3, we show how to solve the 3D imaging problem using Markov random field modeling, loopy belief propagation, and sparse signal processing. We then discuss how to design efficient 3D UAV paths in Section 4. Finally, we present our experimental testbed in Section 5 and our experimental results for 3D through-wall imaging of unknown areas in Section 6, followed by a discussion in Section 7 .

\section{PROBLEM FORMULATION}

Consider a completely unknown area $\mathcal{D} \subset \mathbb{R}^{3}$, which may contain several occluded objects that are not directly visible, due to the presence of walls and other objects in $\mathcal{D} .{ }^{1}$ We are interested in imaging $\mathcal{D}$ using two Unmanned Aerial Vehicles (UAVs) and only WiFi RSSI measurements. Fig. 1 shows two example scenarios, where two

${ }^{1}$ In this paper, we will interchangeably use the terms "domain", "area" and "region" to refer to the $3 \mathrm{D}$ region that is being imaged.
UAVs fly outside of the area of interest, with one transmitting a WiFi signal (TX UAV) and the other one receiving it (RX UAV). In this example, the domain $\mathcal{D}$ would correspond to the walls as well as the region behind the walls.

When the TX UAV transmits a WiFi signal, the objects in $\mathcal{D}$ affect the transmission, leaving their signatures on the collected measurements. Therefore, we first model the impact of objects on the wireless transmissions in this section, and then show how to do 3D imaging and design UAV paths in the subsequent sections. Consider a wireless transmission from the transmitting UAV to the receiving one. Since our goal is to perform 3D imaging based on only RSSI measurements, we are interested in modeling the power of the received signal as a function of the objects in the area. To fully model the receptions, one needs to write the volume-integral wave equations [9], which will result in a non-linear set of equations with a prohibitive computational complexity for our 3D imaging problem. Alternatively, there are simpler linear approximations that model the interaction of the transmitted wave with the area of interest. Wentzel-Kramers-Brillouin (WKB) and Rytov are two examples of such linear approximations [9]. WKB approximation, for instance, only considers the impact of the objects along the line connecting the transmitter (TX) and the receiver (RX). This model is a very good approximation at very high frequencies, such as X-ray, since a wave then primarily propagates along a straight line path, with negligible reflections or diffractions [9]. Rytov approximation, on the other hand, considers the impact of some of the objects that are not along the direct path that connects the TX and RX, at the cost of an increase in computational complexity, and is a good approximation under certain conditions [9].

In this paper, we use a WKB-based approximation to model the interaction of the transmitted wave with the area of interest. While this model is more valid at very high frequencies, several work in the literature have shown its effectiveness when sensing with signals that operate at much lower frequencies such as $\mathrm{WiFi}$ $[12,38]$. WKB approximation can be interpreted in the context of the shadowing component of the wireless channel, as we shall summarize next.

Consider the received power for the $i^{\text {th }}$ signal transmitted from the TX UAV to the RX one. We can express the received power as follows [25, 32]:

$$
P_{\mathrm{R}}\left(\mathbf{p}_{i}, \mathbf{q}_{i}\right)=P_{\mathrm{PL}}\left(\mathbf{p}_{i}, \mathbf{q}_{i}\right)+\gamma \sum_{j} d_{i j} \eta_{i j}+\zeta\left(\mathbf{p}_{i}, \mathbf{q}_{i}\right),
$$


where $P_{\mathrm{R}}\left(\mathbf{p}_{i}, \mathbf{q}_{i}\right)$ denotes the received signal power (in $\mathrm{dB}$ ) for the $i^{\text {th }}$ measurement, when the TX and RX are located at $\mathbf{p}_{i} \in \mathbb{R}^{3}$ and $\mathbf{q}_{i} \in$ $\mathbb{R}^{3}$ respectively. Furthermore, $P_{\mathrm{PL}}\left(\mathbf{p}_{i}, \mathbf{q}_{i}\right)=10 \log _{10} \frac{\beta P_{\mathrm{T}}}{\left(\left\|\mathbf{p}_{i}-\mathbf{q}_{i}\right\|_{2}\right)^{\alpha}}$ is the path loss power (in $\mathrm{dB}$ ), where $P_{\mathrm{T}}$ is the transmit power, $\beta$ is a constant that depends on the system parameters and $\alpha$ is the path loss exponent. ${ }^{2}$ The term $\gamma \sum_{j} d_{i j} \eta_{i j}$ is the shadowing (shadow fading) term in the $\mathrm{dB}$ domain, which captures the impact of the attenuations of the objects on the line connecting the TX and RX UAVs. More specifically, $d_{i j}$ is the distance traveled by the signal within the $j^{\text {th }}$ object along the line connecting the TX and the RX for the $i^{\text {th }}$ measurement, $\eta_{i j}$ is the decay rate of the signal in the $j^{\text {th }}$ object along this line, and $\gamma=10 \log _{10} e$ is a constant. Finally, $\zeta\left(\mathbf{p}_{i}, \mathbf{q}_{i}\right)$ represents the modeling error in this formulation, which includes the impact of multipath fading and scattering off of objects not directly along the line connecting the TX and RX, as well as other un-modeled propagation phenomena and noise. In summary, Eq. 1, which we shall refer to as LOS-based modeling, additively adds the attenuations caused by the objects on the direct line connecting the TX and the RX.

The shadowing term can then be re-written as

$$
\sum_{j} d_{i j} \eta_{i j}=\int_{\mathbb{L}_{\mathbf{p}_{i} \rightarrow \mathrm{q}_{i}}} \eta\left(\mathbf{r}^{\prime}\right) \mathrm{d} \mathbf{r}^{\prime},
$$

where $\int_{\mathbb{L}_{\mathrm{p}_{i} \rightarrow \mathrm{q}_{i}}}$ denotes the line integral along the line connecting the TX and the RX, and $\eta(\mathbf{r})$ denotes the decay rate of the wireless signal at $\mathbf{r} \in \mathcal{D}$. Furthermore, $\eta(\mathbf{r})<0$ when there is an object at position $\mathbf{r}$ and $\eta(\mathbf{r})=0$ otherwise. $\eta$ then implicitly carries information about the area we are interested in imaging.

In order to solve for $\eta$, we discretize $\mathcal{D}$ into $N$ cubic cells of equal volume. Each cell is denoted by its center $\mathbf{r}_{n}$, where $n \in\{1, \ldots, N\}$. By discretizing Eq. 2, we have,

$$
\int_{\mathbb{L}_{\mathbf{p}_{i} \rightarrow \mathrm{q}_{i}}} \eta\left(\mathbf{r}^{\prime}\right) \mathrm{d} \mathbf{r}^{\prime} \approx \sum_{j \in \mathcal{L}\left(\mathbf{p}_{i}, \mathbf{q}_{i}\right)} \eta\left(\mathbf{r}_{j}\right) \Delta d,
$$

where $\mathcal{L}\left(\mathbf{p}_{i}, \mathbf{q}_{i}\right)$ denotes the set of cells along the line connecting the TX and the RX for the $i^{\text {th }}$ measurement, and $\Delta d$ is the dimension of a side of the cubic cell. Therefore, we can approximate Eq. 1 as

$$
P_{i}=\frac{P_{\mathrm{R}}\left(\mathbf{p}_{i}, \mathbf{q}_{i}\right)-P_{\mathrm{PL}}\left(\mathbf{p}_{i}, \mathbf{q}_{i}\right)}{\gamma \Delta d} \approx \sum_{j \in \mathcal{L}\left(\mathbf{p}_{i}, \mathbf{q}_{i}\right)} \eta\left(\mathbf{r}_{j}\right),
$$

with $P_{i}$ denoting the normalized received power of the $i^{\text {th }}$ measurement. By stacking the measurements $P_{i}$ as a column vector, we have,

$$
\mathrm{P} \approx \mathrm{AO},
$$

where $\mathbf{P}=\left[P_{1}, P_{2}, \ldots, P_{M}\right]^{T}$ and $M$ is the number of measurements. A is a matrix of size $M \times N$ such that its entry $A_{i, j}=1$ if the $j^{\text {th }}$ cell is along the line connecting the TX and the RX for the $i^{\text {th }}$ measurement, and $A_{i, j}=0$ otherwise. Furthermore, $\mathbf{O}=\left[\eta\left(\mathbf{r}_{1}\right), \eta\left(\mathbf{r}_{2}\right), \ldots, \eta\left(\mathbf{r}_{N}\right)\right]^{T}$ represents the property of objects in the area of interest $\mathcal{D}$, which we shall refer to as the object map.

\footnotetext{
${ }^{2}$ In practice, the two parameters of the path loss component can be estimated by using a few line-of-sight transmissions between the two UAVs, near the area of interest when there are no objects in between them.
}

So far, we have described the system model that relates the wireless measurements to the object map, which contains the material properties of the objects in the area of interest. In this paper, we are interested in imaging the geometry and locations of all the objects in $\mathcal{D}$, as opposed to characterizing their material properties. More specifically, we are interested in obtaining a binary object map $\mathbf{O}_{b}$ of the domain $\mathcal{D}$, where $\mathbf{O}_{b}$ is a vector whose $i^{\text {th }}$ element is defined as follows:

$$
O_{b_{i}}=\left\{\begin{array}{ll}
1 & \text { if the } i^{\text {th }} \text { cell contains an object } \\
0 & \text { otherwise }
\end{array} .\right.
$$

In the next sections, we propose to estimate $\mathbf{O}_{b}$ by first solving for $\mathbf{O}$ and then making a decision about the presence or absence of an object at each cell, based on the estimated $\mathrm{O}$, using loopy belief propagation.

\section{SOLVING THE 3D IMAGING PROBLEM}

In the previous section, we formulated the problem of reconstructing the object map as a system of linear equations, with the final goal of imaging a binary object map of the domain $\mathcal{D}$. In this section, we propose a two-step approach for $3 \mathrm{D}$ imaging of $\mathrm{O}_{b}$. In the first part, we utilize techniques from the sparse signal processing and regularization literature to solve Eq. 5, and thereby estimate O. In the second part, we use loopy belief propagation in order to image a binary object map $\mathbf{O}_{b}$ based on the estimated $\mathrm{O}$. We note that in some of the past literature on $2 \mathrm{D}$ imaging $[8,12]$, either the estimated object map is directly thresholded to form a binary image, or the grayscale image is considered as the final image. Since 3D imaging with only WiFi signals becomes a considerably more challenging problem, such approaches do not suffice anymore. Instead, we propose to use loopy belief propagation in order to obtain the final 3D image, as we shall see later in this paper.

\subsection{Sparse Signal Processing}

In this part, we aim to solve for $\mathrm{O}$ in Eq. 5. In typical practical cases, however, $N \gg M$, i.e., the number of wireless measurements is typically much smaller than the number of unknowns, which results in a severely under-determined underlying system. Then, if no additional condition is imposed, there will be a considerable ambiguity in the solution. We thus utilize the fact that several common spaces are sparse in their spatial variations, which allows us to borrow from the literature on sparse signal processing. Sparse signal processing techniques aim at solving an under-determined system of equations when there is an inherent sparsity in the signal of interest, and under certain conditions on how the signal is sampled $[7,15]$. They have been heavily utilized in many different areas and have also proven useful in the area of sensing with radio frequency signals (e.g., 2D imaging, tracking) [17, 23, 31]. Thus, we utilize tools from sparse signal processing to estimate $\mathbf{O}$, the map of the material properties. This estimated map will then be the base for our 3D imaging approach in the next section.

More specifically, we utilize the fact that most areas are sparse in their spatial variations and seek a solution that minimizes the Total Variation (TV) of the object map O. We next briefly summarize our 3D TV minimization problem, following the notation in [26]. 
As previously defined, $\mathbf{O}$ is a vector representing the map of the objects in the domain $\mathcal{D}$. Let $\mathrm{I}$ be the 3D matrix that corresponds to $\mathbf{O}$. $\mathbf{I}$ is of dimensions $n_{1} \times n_{2} \times n_{3}$, where $N=n_{1} \times n_{2} \times n_{3}$. We seek to minimize the spatial variations of I, i.e., for every element $I_{i, j, k}$ in $\mathbf{I}$, the variations across the three dimensions need to be minimized. Let $D_{m} \in \mathbb{R}^{3 \times N}$ denote a matrix such that $D_{m} \mathrm{O}$ is a $3 \times 1$ vector of the spatial variations of the $m^{\text {th }}$ element in $\mathbf{O}$, with $m$ corresponding to the $(i, j, k)^{\text {th }}$ element in I. The structure of $D_{m}$ is such that $D_{m} \mathrm{O}=\left[I_{i+1, j, k}-I_{i, j, k}, I_{i, j+1, k}-I_{i, j, k}, I_{i, j, k+1}-I_{i, j, k},\right]^{T}$. Then, the TV function is given by

$$
\operatorname{TV}(\mathbf{O})=\sum_{i=1}^{N}\left\|D_{i} \mathbf{O}\right\|_{2},
$$

where $\|.\|_{2}$ denotes the $l_{2}$ norm of the argument. We then have the following TV minimization problem:

$$
\text { minimize } \operatorname{TV}(\mathbf{O}) \text {, subject to } \mathbf{P}=\mathrm{AO} \text {, }
$$

where $\mathbf{P}, \mathbf{A}$ and $\mathbf{O}$ are as defined in Eq. 5.

In order to solve the 3D TV minimization problem of Eq. 8, an efficient practical implementation using Nesterov's algorithm, TVReg has been proposed in [26]. TVReg is a MATLAB-based solver that efficiently computes the 3D TV minimization solution. We use TVReg for solving the optimization problem of Eq. 8 in all the results of the paper.

The solution obtained from solving Eq. 8 is an approximation to the object map $\mathbf{O}$. As previously mentioned in Section 2, the elements of $\mathrm{O}$ are all non-positive real numbers. We then flip the sign and normalize the values to the range $[0,1]$, so that they represent the grayscale intensities at the corresponding cells, which we denote by $\mathbf{y}_{s}$. However, this solution is not a perfect representation of the object map, due to modeling errors and the under-determined nature of the linear system model, requiring further processing. Furthermore, we are only interested in estimating the presence or absence of an object at any location, as opposed to learning the material properties in this paper. Therefore, we next describe our approach for estimating the binary object map $\mathbf{O}_{b}$ of the domain $\mathcal{D}$, given the observed intensities $\mathbf{y}_{s}$.

\subsection{D Imaging Using Loopy Belief Propagation}

In this section, we consider the problem of estimating the 3D binary image of the unknown domain $\mathcal{D}$, based on the solution $\mathbf{y}_{s}$ of the previous section. As discussed earlier, $\mathbf{y}_{s}$ can be interpreted as the estimate of the gray-scale intensities at the cells in the 3D space. We are then interested in estimating the 3D binary image, which boils down to finding the best labels (occupied/not occupied) for each cell in the area of interest, while minimizing the impact of modeling errors/noise and preserving the inherent spatial continuity of the area.

To this end, we model the 3D binary image as a Markov Random Field (MRF) [6] in order to capture the spatial dependencies among local neighbors. Using the MRF model, we can then use the Hammersley-Clifford Theorem to express the probability distribution of the labels in terms of locally-defined dependencies. We then show how to estimate the binary occupancy state of each cell in the 3D domain, by using loopy belief propagation [6] on the defined
MRF. Utilizing loopy belief propagation provides a computationallyefficient way of solving the underlying optimization problem, as we shall see. We next describe the details of our approach.

Consider a random vector $\mathrm{X}$ that corresponds to the binary object map $\mathbf{O}_{b}$. Each element $X_{i} \in\{0,1\}$ is a random variable that denotes the label of the $i^{\text {th }}$ cell. Further, let $\mathrm{Y}$ denote a random vector representing the observed grayscale intensities. In general, there exists a spatial continuity among neighboring cells of an area. An MRF model accounts for such spatial contextual information, and is thus widely used in the image processing and vision literature for image denoising, image segmentation, and texture labeling [16, 22], as we discussed earlier. We next formally define an MRF.

Definition 3.1. A random field $\mathbf{U}$ on a graph is defined as a Markov Random Field (MRF) if it satisfies the following condition: $P\left(U_{i}=\right.$ $\left.u_{i} \mid U_{j}=u_{j}, \forall j \neq i\right)=P\left(U_{i}=u_{i} \mid U_{j}=u_{j}, \forall j \in \mathcal{N}_{i}\right)$, where $\mathcal{N}_{i}$ is the set of the neighboring nodes of $i$.

In summary, every node is independent of the rest of the graph in an MRF, when conditioned on its neighbors. This is a good assumption for the 3D areas of interest to this paper. We thus next model our underlying system as an MRF. Consider the graph $\mathcal{G}=(\mathcal{V}, \mathcal{E})$ corresponding to a 3D discrete grid formed from the cells in the domain, where $\mathcal{V}=\{1,2, \ldots, N\}$ is the set of nodes in the graph. Each node $i$ is associated with a random variable $X_{i}$, that specifies the label assigned to that node. Furthermore, the edges of the graph $\mathcal{E}$ define the neighborhood structure of our MRF. In this paper, we assume that each node in the interior of the graph is connected via an edge to its 6 nearest neighbors in the 3D graph, as is shown in Fig. 2. Additionally, since $\mathrm{X}$ is unobserved and needs to be estimated, all the nodes associated with $\mathrm{X}$ are referred to as hidden nodes [6]. Furthermore, $Y_{i}$ is the observation of the hidden node $i$. These observations are typically modeled as being independent when conditioned on the hidden variables [6]. More specifically, the observations are assumed to satisfy the following property: $P(\mathrm{Y}=\mathbf{y} \mid \mathbf{X}=\mathbf{x})=\prod_{i} P\left(Y_{i}=y_{i} \mid X_{i}=x_{i}\right)$. This is a widely-used assumption in the image processing and computer vision literature [6], where the observations correspond to the observed intensities at the pixels. We adopt this model for our scenario by adding a new set of nodes called the observed nodes to our graph $\mathcal{G}$. Each observed node $Y_{i}$ is then connected by an edge to the hidden node $X_{i}$. Fig. 2 shows our described graph structure, where all the 6 hidden neighbors and an additional observed neighbor are shown for a node in the interior of the graph. For the nodes at the edge of the graph, the number of hidden node neighbors will be either 3, 4 or 5 , depending on their position.

The advantage of modeling the 3D image as an MRF is that the joint probability distribution of the labels over the graph can be solely expressed in terms of the neighborhood cost functions. This result follows from the Hammersley-Clifford theorem [5], which we summarize next.

THEOREM 3.2. Suppose that $\mathbf{U}$ is a random field defined over a graph, with a joint probability distribution $P(\mathbf{U}=\mathbf{u})>0$. Then, $\mathbf{U}$ is a Markov Random Field if and only if its joint probability distribution is given by $P(\mathbf{U}=\mathbf{u})=\frac{1}{Z} \exp (-E(\mathbf{u}))$, where $E(\mathbf{u})=\sum_{c \in C} \Phi_{c}\left(u_{c}\right)$ is the energy or cost associated with the label $\mathbf{u}$ and $Z=\sum_{\mathbf{u}} \exp (-E(\mathbf{u}))$ is a normalization constant. Further, $C$ is the set of all the cliques in 


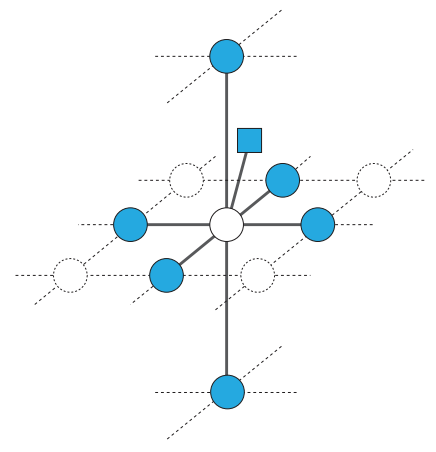

Figure 2: A depiction of the six-connected neighborhood structure of the underlying graph that corresponds to the Markov Random Field modeling of our 3D area of interest Each node in the interior of the graph has six hidden nodes and one observed node as neighbors. The shaded circular nodes denote the neighbors that correspond to the hidden nodes, and the shaded square represents the observed node.

the graph, $\Phi_{c}\left(u_{c}\right)$ is the cost associated with the clique $c$, and $u_{c}$ is the realization (labels) associated with the nodes in $c^{3}{ }^{3}$

Proof. See [5] for details.

We next establish that our defined graph of hidden and observed nodes is an MRF and thus satisfies the joint distribution of Theorem 3.2. More specifically, based on our defined neighborhood system, every hidden node $X_{i}$ in the interior of the graph has a neighborhood of six hidden nodes and one observed node. Furthermore, every observed node $Y_{i}$ has one neighbor, the corresponding hidden node $X_{i}$, as we established. Let $U_{i}$ denote any node in this graph, which can correspond to a hidden or an observed node. Such a node $U_{i}$ is independent of the rest of the graph, when conditioned on its neighbors. Therefore, the overall graph consisting of hidden and observed nodes is an MRF. Then, by using the Hammersley-Clifford Theorem (Theorem 3.2), we get the following joint probability distribution for the nodes,

$$
P(\mathbf{X}=\mathbf{x}, \mathbf{Y}=\mathbf{y})=\frac{1}{Z} \exp (-E(\mathbf{x}, \mathbf{y})),
$$

where $Z=\sum_{\mathbf{x}, \mathbf{y}} \exp (-E(\mathbf{x}, \mathbf{y}))$ is a normalization constant, and $E(\mathbf{x}, \mathbf{y})$ is defined over the cliques of the graph. In our case, the graph has cliques of size 2. Furthermore, there are two kinds of cliques in the graph: cliques associated with two hidden nodes and cliques associated with one hidden and one observed node. Therefore, $E(\mathbf{x}, \mathbf{y})$ can be expressed as follows:

$$
E(\mathbf{x}, \mathbf{y})=\sum_{i=1}^{N} \Phi_{i}\left(x_{i}, y_{i}\right)+\sum_{(i, j) \in \mathcal{E}} \Phi_{i j}\left(x_{i}, x_{j}\right)
$$

In the above equation, $\Phi_{i}\left(x_{i}, y_{i}\right)$ is the cost of associating a label $x_{i}$ to a hidden node that has a corresponding observation $y_{i}$. Furthermore, $\Phi_{i j}\left(x_{i}, x_{j}\right)$ is the cost of associating label $\left(x_{i}, x_{j}\right)$ to a neighboring pair of hidden nodes $(i, j)$.

\footnotetext{
${ }^{3} \mathrm{~A}$ clique in a graph is defined as a set of nodes that are completely connected.
}

Given a set of observations $\mathbf{y}_{s}$, we then consider finding the $\mathbf{x}$ (labels) that maximizes the posterior probability (MAP), i.e., $P(\mathrm{X}=$ $\mathbf{x} \mid \mathbf{Y}=\mathbf{y}_{s}$ ). From Eq. 9, we have,

$$
P\left(\mathbf{X}=\mathbf{x} \mid \mathrm{Y}=\mathbf{y}_{s}\right)=\frac{1}{Z_{y}} \exp \left(-E\left(\mathbf{x}, \mathbf{y}_{s}\right)\right)
$$

where $Z_{y}=\sum_{\mathbf{x}} \exp \left(-E\left(\mathbf{x}, \mathbf{y}_{s}\right)\right)$ is a normalization constant. It then follows from Theorem 3.2 and Eq. 11 that $\mathrm{X}$ given $\mathrm{Y}=\mathrm{y}_{s}$ is also an MRF over the graph $\mathcal{G}$ of the hidden variables defined earlier.

However, directly solving for $\mathbf{x}$ that maximizes Eq. 11 is combinatorial and thus computationally prohibitive. Several distributed and iterative algorithms have thus been proposed in the literature to efficiently solve this classical problem of inference over a graph [28]. Belief propagation is one such algorithm, which has been extensively used in the vision and channel coding literature [6, 37]. In this paper, we then utilize belief propagation to efficiently solve the problem of estimating the best labels over the graph, given the observations $\mathrm{y}_{s}$.

3.2.1 Utilizing Loopy Belief Propagation.

Belief propagation based algorithms can find the optimum solution for graphs without loops, but provide an approximation for graphs with loops. ${ }^{4}$ In our case, the graph representing our inference problem of interest has loops, which is a common trend for graphs representing vision and image processing applications. Even though belief propagation is an approximation for graphs with loops, it is shown to provide good results in the literature [37].

There are two versions of the belief propagation algorithm: the sum-product and the max-product. The sum-product computes the marginal distribution at each node, and estimates a label that maximizes the corresponding marginal. Thus, this approach finds the best possible label for each node individually. On the other hand, the max-product approach computes the labels that maximize the posterior probability (MAP) over the entire graph. Thus, if the graph has no loops, the max-product approach converges to the solution of Eq. 11, which is the optimum solution.

Loopy belief propagation refers to applying the belief propagation algorithms to the graphs with loops. In such cases, there is no guarantee of convergence to the optimum solution for the maxproduct or sum-product methods. However, several work in the literature have used these two methods with graphs with loops and have shown good results $[16,34,40]$. In this paper, we thus utilize the sum-product version, which has better convergence guarantees [37], to estimate the labels of the hidden nodes. We next describe the sum-product loopy belief propagation algorithm [39].

The sum-product loopy belief propagation is a message passing algorithm that computes the marginal of the nodes in a distributed manner. Let $m_{i j}^{(t)}\left(x_{j}\right)$ denote the message that node $i$ passes to node $j$, where $t$ denotes the iteration number. The update rule for the messages is given by

$$
m_{i j}^{(t)}\left(x_{j}\right)=\lambda_{m} \sum_{x_{i}} \Psi_{i}\left(x_{i}, y_{i}\right) \Psi_{i j}\left(x_{i}, x_{j}\right) \prod_{k \in \mathcal{N}_{i} \backslash j} m_{k i}^{(t-1)}\left(x_{i}\right),
$$

${ }^{4}$ In a graph with loops, solving for the optimal set of labels is an NP-hard problem [35]. 
where $\Psi_{i}\left(x_{i}, y_{i}\right)=\exp \left(-\Phi_{i}\left(x_{i}, y_{i}\right)\right)$ corresponds to the observation dependency, $\Psi_{i j}\left(x_{i}, x_{j}\right)=\exp \left(-\Phi_{i j}\left(x_{i}, x_{j}\right)\right)$ corresponds to the spatial dependency, $\mathcal{N}_{i}$ denotes the set of neighbors of node $i$ in $\mathcal{G}$ and $\lambda_{m}$ is a normalization constant. The belief (marginal) at each node is then calculated by

$$
b_{i}^{(t)}\left(x_{i}\right)=\lambda_{b} \Psi_{i}\left(x_{i}, y_{i}\right) \prod_{k \in \mathcal{N}_{i}} m_{k i}^{(t)}\left(x_{i}\right),
$$

where $\lambda_{b}$ is a normalization constant. Finally, after the algorithm converges, the final solution (labels) $\hat{\mathbf{x}}$ is calculated at each node as follows:

$$
\hat{x}_{i}=\arg \max _{x_{i}} b_{i}\left(x_{i}\right) .
$$

The algorithm starts with the messages initialized at one. A stopping criteria is then imposed by setting a threshold on the average changes in the belief of the nodes, and a threshold on the maximum number of iterations. The final solution is then the estimated $\mathbf{O}_{b}$, i.e., the 3D binary image of the area of interest.

\subsubsection{Defining the Cost Functions.}

We next define the $\Phi_{i}$ and $\Phi_{i j}$ that we shall utilize as part of our loopy belief propagation algorithm of Eq. 10 and 12. Based on the cost functions chosen in the image restoration literature [16], we choose $\Phi_{i j}\left(x_{i}, x_{j}\right)=\left(x_{i}-x_{j}\right)^{2}$ and $\Phi_{i}\left(x_{i}, y_{i}\right)=\left(x_{i}-y_{i}\right)^{2}$. In several cases, the outer edge of the area of interest, e.g., the pixels corresponding to the outer most layer of the boundary wall, can be sensed with other sensors such as a camera or a laser scanner. In such cases, we can then modify $\Phi_{i}\left(x_{i}, y_{i}\right)$ as follows to enforce this information: $\Phi_{i}\left(x_{i}, y_{i}\right)=\left\{\begin{array}{ll}\left(1-x_{i}\right) & \text { if } i \in \Omega_{B} \\ \left(x_{i}-y_{i}\right)^{2} & \text { otherwise }\end{array}\right.$, where $\Omega_{B}$ denotes the set of graph nodes that constitute the outer boundary of the domain.

In summary, the solution $\hat{\mathbf{x}}$ that we obtain from the loopy belief propagation algorithm is the estimate of $\mathrm{O}_{b}$, which is our 3D binary image of the area of interest.

\section{UAV PATH PLANNING}

So far, we have described the system model and the proposed approach for solving the $3 \mathrm{D}$ through-wall imaging problem, given a set of wireless measurements. The TX/RX locations where the measurements are collected can play a key role in the 3D imaging quality. By using unmanned aerial vehicles, we can properly design and control their paths, i.e., optimize the locations of the TX/RX, in order to autonomously and efficiently collect the measurements that are the most informative for 3D imaging, something that would be prohibitive with fixed sensors. In this section, we discuss our approach for planning efficient and informative paths for 3D imaging with the UAVs. We start by summarizing the state-of-the-art in path planning for 2D imaging with ground vehicles [19]. We then see why the $2 \mathrm{D}$ approach can not be fully extended to $3 \mathrm{D}$, which is the main motivation for designing paths that are efficient and informative for 3D imaging with UAVs.

In [19], the authors have shown the impact of the choice of measurement routes on the imaging quality for the case of $2 \mathrm{D}$ imaging with ground vehicles. Let the spatial variations along a given direction be defined as the variations of the line integral described in Eq. 2, when the TX and RX move in parallel along

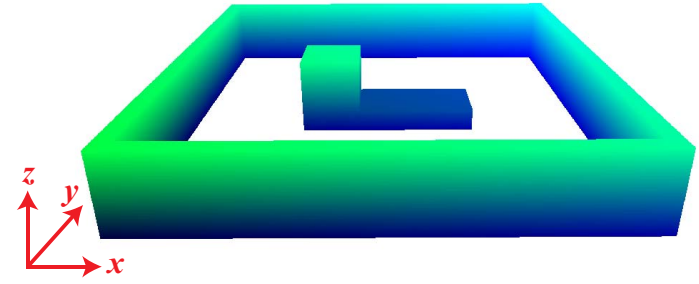

Figure 3: An example scenario with an L-shaped structure located behind the walls.

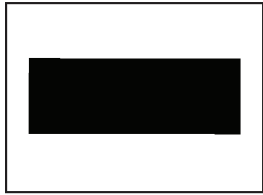

(a)

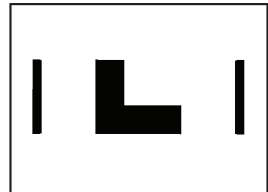

(b)

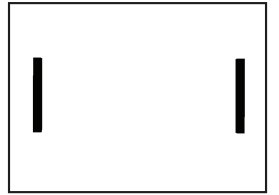

(c)
Figure 4: 2D cross sections corresponding to three $x-z$ planes at different $y$ coordinates for the area of Fig. 3. As can be seen, the information about the variations in the $\mathrm{z}$ direction is only observable in (b).

that direction outside of the area of interest [12, 19]. Fig. 5, for example, marks the $0^{\circ}$ and $45^{\circ}$ directions for a $2 \mathrm{D}$ scenario. We then say that the two vehicles make parallel measurements along the $45^{\circ}$ route if the line that connects the positions of the TX and RX stays orthogonal to the $45^{\circ}$ line that passes through the origin. ${ }^{5}$ Then, for every TX/RX position pair along this route, we evaluate the line integral of Eq. 2 and define the spatial variations along this direction as the variations of the corresponding line integral. Furthermore, let the jump directions be defined as those directions of measurement routes along which there exist most abrupt spatial variations.

For the case of 2D imaging using unmanned ground vehicles, the authors in [19] have shown that one can obtain good imaging results by using parallel measurement routes at diverse enough angles to capture most of the jumps. Since in a horizontal 2D plane, there are typically only a few major jump directions, then measurements along a few parallel routes that are diverse enough in their angles can suffice for 2D imaging. For instance, as a toy example, consider the area of interest of Fig. 3. For the 2D imaging of a horizontal cut of this area, we only need to choose a few diverse angles for the parallel routes in a constant $\mathrm{z}$ plane.

Next, consider the whole 3D area of Fig. 3. The measurements that are collected on parallel routes along the jump directions would still be optimal in terms of imaging quality. However, collecting such measurements can become prohibitive, as it requires additional parallel routes in many $x-z$ or $y-z$ planes. This is due to the fact that the added dimension can result in significant spatial variations along all three directions in 3D. For instance, in order to obtain

\footnotetext{
${ }^{5}$ We note that such routes are sometimes referred to as semi-parallel routes in the literature, as opposed to parallel routes, since the two vehicles do not have to go in parallel. Rather, the line connecting the two needs to stay orthogonal to the line at the angle of interest. For the sake of simplicity, we refer to these routes as parallel routes in this paper.
} 


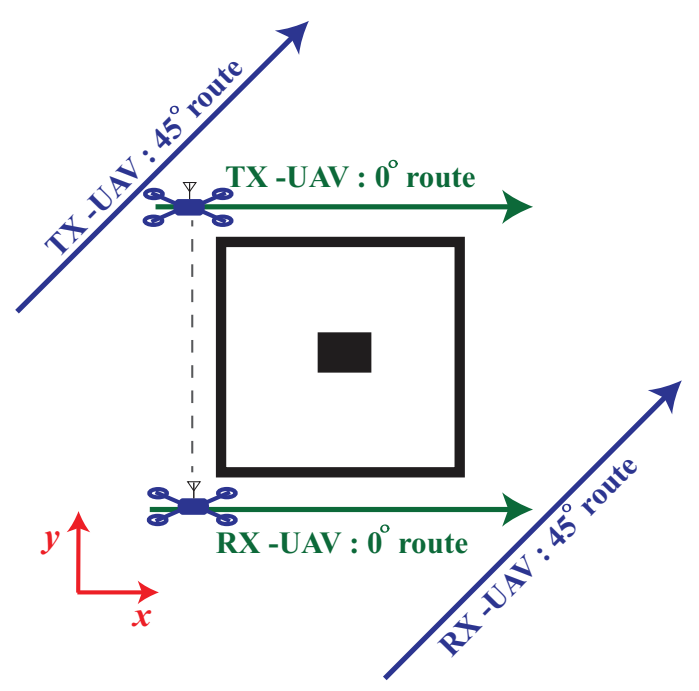

Figure 5: An illustration showing the projection of the proposed routes onto the $x-y$ plane. The routes corresponding to $0^{\circ}$ and $45^{\circ}$ are shown as examples.

information about the jumps in the $\mathrm{z}$ direction in Fig. 3, one would need to design additional parallel routes in various $x-z$ or $y$-z planes. However, there exist many such planes that will not provide any useful information about the unknown domain. For instance, Fig. 4 shows three $\mathrm{x}-\mathrm{z}$ plane cross-sections for the area of Fig. 3. As can be seen, only the plane corresponding to Fig. 4 (b) would provide valuable information about the jumps in the $\mathrm{z}$ direction. Therefore, a large number of parallel measurements along $x-y, x-z$, or $y-z$ planes are required to capture useful information for 3D imaging.

In summary, since the jump directions are now distributed over various planes, it can become more challenging to collect informative measurements unless prohibitive parallel measurements in many $x-y, x-z$, or $y-z$ planes are made. We then propose a path planning framework that would efficiently sample the unknown domain, so that we obtain information about the variations in the $\mathrm{z}$ direction as well as the variations in $x-y$ planes, without directly making several parallel routes in $\mathrm{x}-\mathrm{z}$ or $\mathrm{y}-\mathrm{z}$ planes. More specifically, in order to efficiently capture the changes in all the three dimensions, we use two sets of parallel routes, as described below:

(1) In order to capture the variations in the $x-y$ directions, we choose a number of constant $\mathrm{z}$ planes and make a diverse set of parallel measurements, as is done in 2D. Fig. 5 shows sample such directions at $0^{\circ}$ and $45^{\circ}$.

(2) In order to capture the variations in the $z$ direction, we then use sloped routes in a number of planes, two examples of which are shown in Fig. 6. More specifically, for a pair of parallel routes designed in the previous item for $2 \mathrm{D}$, consider a similar pair of parallel routes with the same $\mathrm{x}$ and $\mathrm{y}$ coordinates for the TX and RX, but with the $\mathrm{z}$ coordinate defined as $z=a \delta+b$, where $\delta$ is the distance traveled along the route when projected to a $2 \mathrm{D} x-y$ plane, and $a$ and $b$ are constants defining the corresponding line in $3 \mathrm{D}$. We refer to such a route as a sloped route, and the corresponding plane (that contains two such parallel routes

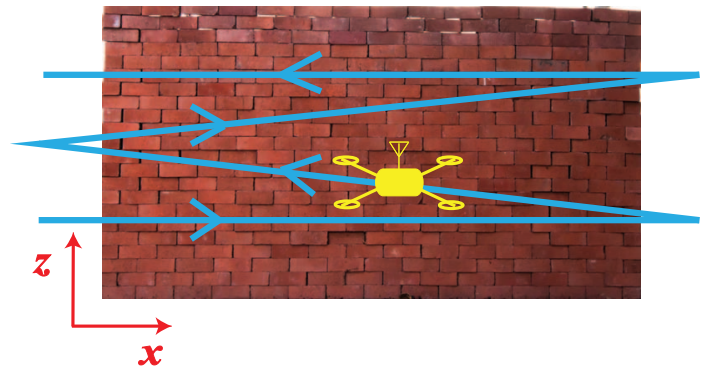

Figure 6: Example routes corresponding to two horizontal and two sloped routes for one UAV. The other UAV is on the other side of the domain at the corresponding parallel locations.

traveled in parallel by two UAVs) as a sloped plane. Fig. 5 can then also represent the projection of the parallel routes of the sloped planes onto the $\mathrm{x}-\mathrm{y}$ plane as well.

Fig. 6 shows an example of these two types of routes, for one UAV, along two horizontal and two sloped routes. For each route, the other UAV will traverse the corresponding parallel route on the other side of the structure. When projected to the $z=0$ plane, all the depicted routes will correspond to $\theta=0^{\circ}$ route of Fig. 5 in this example.

In summary, while designing parallel routes along $\mathrm{x}-\mathrm{z}$ or $\mathrm{y}-\mathrm{z}$ planes can directly capture the changes in the $\mathrm{z}$ direction, the sloped routes can also be informative for capturing the variations in the $\mathrm{z}$ direction while reducing the burden of navigation and sampling considerably.

\section{EXPERIMENTAL TESTBED}

In this section, we describe our experimental testbed that enables 3D through-wall imaging using only WiFi RSSI and UAVs that collect wireless measurements along their paths. Many challenges arise when designing such an experimental setup for imaging throughwalls with UAVs. Examples include the need for accurate localization, communication between UAVs, coordination and autonomous route control. We next describe our setup and show how we address the underlying challenges.

\begin{tabular}{|c|c|}
\hline Component & Model/specifications \\
\hline \hline UAV & 3DR X8 octo-copter [1] \\
\hline WiFi router & D-Link WBR 1310 \\
\hline WLAN card & TP-LINK TL-WN722N \\
\hline Localization device & Google Tango Tablet [20] \\
\hline & $16 \mathrm{dBi}$ gain Yagi antenna \\
Directional antenna & $23^{\circ}$ vertical beamwidth \\
& $26^{\circ}$ horizontal beamwidth \\
\hline Raspberry Pi & Raspberry Pi 2 Model B \\
\hline
\end{tabular}

Table 1: List of the components of our experimental setup and their corresponding specifications. 


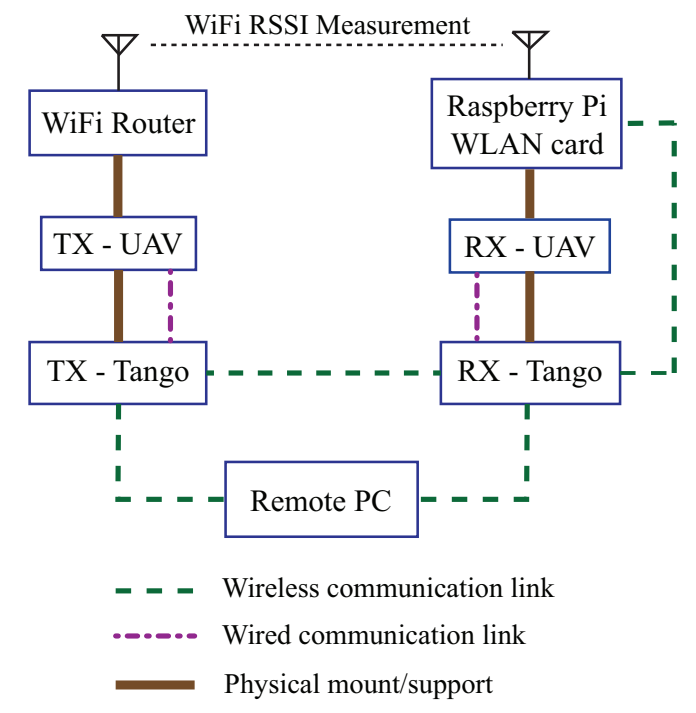

Figure 7: A high-level block diagram of the experimental components and their interactions.

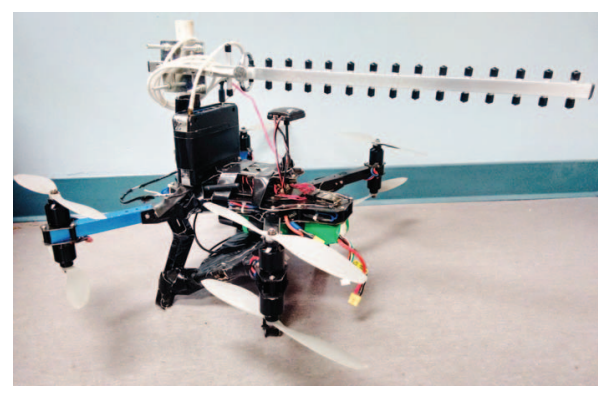

Figure 8: A 3DR X8 octo-copter used in our experiments.

Table 1 shows the specifications of the components that we use in our experiments. The details of how each component is used will be described in the following sections. Fig. 7 shows the overall block diagram of all the components and their interactions. We next describe the details of the experimental components.

\subsection{Basic UAV Setup}

We use two 3DR X8 octo-copters [1] in our experiments. Fig. 8 shows one of our octo-copters. Each UAV has an on-board Pixhawk module, which controls the flight of the UAV. The Pixhawk board receives information about the flight from a controller (e.g., manual controller, auto-pilot or other connected devices), and regulates the motors to control the flight based on the received information. We have further added various components to this basic setup, as described next.

\subsection{Localization}

Localization is a crucial aspect of our experimental testbed. In order to image the unknown region, the UAVs need to put a position stamp on the TX/RX locations where each wireless measurement is collected. Furthermore, the UAVs need to have a good estimate of their position for the purpose of path planning. However, UAVs typically use GPS for localization, the accuracy of which is not adequate for high quality imaging. Therefore, we utilize Google Tango Tablets [20] to obtain localization information along the routes. The Tangos use various on-board cameras and sensors to localize themselves with a high precision in $3 \mathrm{D}$, and hence have been utilized for robotic navigation purposes [30]. In our setup, one Tango is mounted on each UAV. It then streams its localization information to the Pixhawk through a USB port that connects to the serial link of the Pixhawk. The Tango sends information to the Pixhawk using an android application that we modified based on open source $\mathrm{C}++$ and Java code repositories [21,24]. The Pixhawk then controls the flight of the UAVs based on the location estimates. Based on several tests, we have measured the MSE of the localization error (in meters) of the Tango tablets to be 0.0045 .

\subsection{Route Control and Coordination}

The UAVs are completely autonomous in their flight along a route. Each Tango initially receives the route information and way-points (short-term position goals) from the remote PC at the beginning of the route. These way-points are equally-spaced position goals located along the route. In our experiments, the projections of these way-points onto the $x-y$ plane are spaced $5 \mathrm{~cm}$ apart. During the flight, each Tango uses its localization information to check if it has reached the current way-point along its route (within a desired margin of accuracy). If it has reached its own way-point, it then checks if the other Tango has reached the corresponding way-point along its route. If the other Tango indicates that it has not reached its current way-point, then the first Tango waits until the other Tango reaches its desired way-point. Once the Tangos are coordinated, each Tango sends information about the next waypoint to its corresponding Pixhawk. The Pixhawk then controls the flight of the UAV so that it moves towards the next way-point. As a result, both the UAVs are coordinated with each other while moving along their respective routes.

\subsection{WiFi RSSI Measurements}

We next describe our setup for collecting WiFi RSSI measurements. A WiFi router is mounted on the TX UAV, and a WLAN card is connected to a Raspberry Pi, which is mounted on the RX UAV. The WLAN card enables WiFi RSSI measurements, and the Raspberry Pi stores this information during the route, which is then sent to the RX Tango upon the completion of the route. In our experiments, the RX UAV measures the RSSI every $2 \mathrm{~cm}$. More specifically, the RX Tango periodically checks if it has traveled $2 \mathrm{~cm}$ along the route from the previous measurement location, when projected onto the $\mathrm{x}-\mathrm{y}$ plane. If the RX Tango indicates that it has traveled 2 $\mathrm{cm}$, then it records the current localization information of both the Tangos, and communicates with the Raspberry Pi to record an RSSI measurement. At the end of the route, we then have the desired RSSI measurements along with the corresponding positions of the TX and RX UAVs. Finally, in order to mitigate the effect of multipath, directional antennas are mounted on both the TX and RX UAVs for WiFi signal transmission and reception. The specifications of the directional antennas are described in Table 1. 

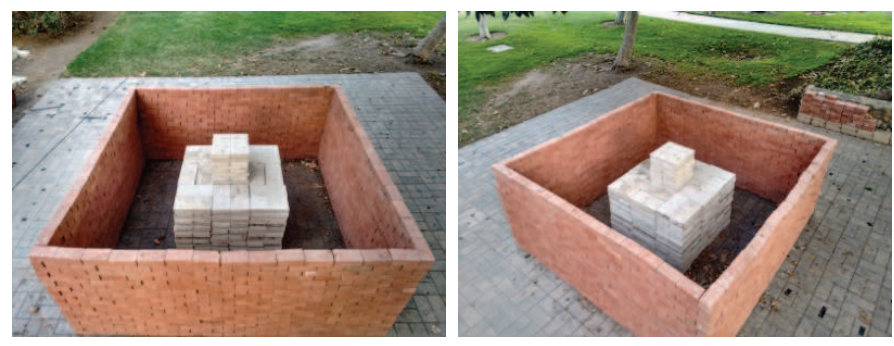

(a)
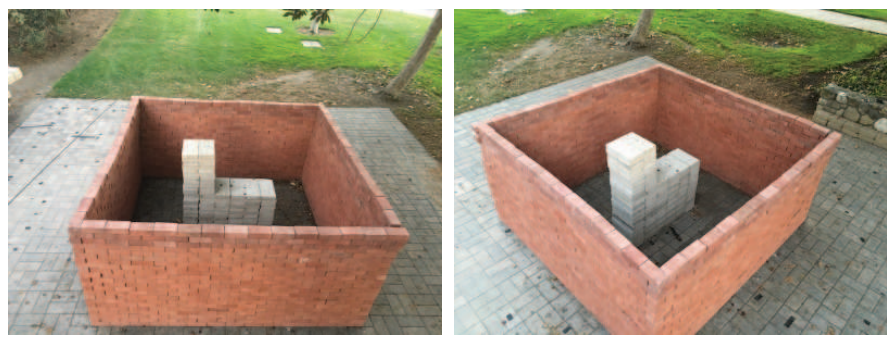

(b)

Figure 9: The two areas of interest for 3D through-wall imaging. (a) shows the two-cube scenario and (b) shows the L-shape scenario. For better clarity, two views are shown for each area.

\section{EXPERIMENTAL RESULTS}

In this section, we first show the results of our proposed framework for 3D through-wall imaging, and then compare our proposed approach with the state-of-the-art in robotic 2D through-wall imaging using WiFi. We use our experimental testbed of Section 5 in order to collect WiFi RSSI measurements outside an unknown area. The area is then reconstructed in 3D based on the approach described in Section 3. In this section, we consider the two areas shown in Fig. 9. We refer to the areas of Fig. 9 (a) and Fig. 9 (b) as the two-cube and L-shape respectively, in reference to the shapes of the structures behind the walls. For both areas, the unknown domain that we image consists of both the outer walls and the enclosed region.

\section{Implementation Details}

We first discuss the specific details of our experiments. The dimensions of the unknown areas to be imaged are $2.96 \mathrm{~m} \times 2.96 \mathrm{~m} \times 0.4$ $\mathrm{m}$ for the two-cube scenario, and $2.96 \mathrm{~m} \times 2.96 \mathrm{~m} \times 0.5 \mathrm{~m}$ for the L-shape scenario. ${ }^{6}$ Each WiFi RSSI measurement recorded by the RX-UAV is an average of 10 samples collected at the same position. A median filter is used on the RSSI measurements to remove spurious impulse noises in the measured data. The routes are chosen according to the design described in Section 4. For capturing the variations in the $\mathrm{x}-\mathrm{y}$ directions, two horizontal planes are chosen. The first horizontal plane is at a height of $5 \mathrm{~cm}$ above the lower boundary of the area to be imaged, while the second horizontal plane is at a height of $5 \mathrm{~cm}$ below the upper boundary of the area to be imaged. In each of these planes, parallel routes are taken with their directions corresponding to $\left\{0^{\circ}, 45^{\circ}, 90^{\circ}, 135^{\circ}\right\}$ (see Fig. 5 for examples of $0^{\circ}$ and $45^{\circ}$ ). Additionally, for every pair of such parallel routes, there are two corresponding pairs of sloped routes as defined in Section 4 (z coordinate varying as $z=a \delta+b$ ), with $0.2 / D$ representing the slope of each sloped route, where $D$ is the total distance of the route when projected to the $\mathrm{x}-\mathrm{y}$ plane, 0.2 corresponds to the total change in height along one sloped route, and the offset $b$ is such that the intersection of the sloped routes shown in Fig. 6 corresponds to the height of the mid-point of the area to be imaged. This amounts to the total of eight sloped routes and eight horizontal routes, four of which are shown in Fig. 6.

\footnotetext{
${ }^{6}$ The area to be imaged does not start at the ground, but at a height of $0.65 \mathrm{~m}$ above the ground. This is because the Tangos need to be at least $0.35 \mathrm{~m}$ above the ground for a proper operation and the antenna mounted on the UAV is at a height of $0.3 \mathrm{~m}$ above the Tango. Also, note that the UAVs fly well below the top edge of the walls, and therefore do not have any visual information about the area inside.
}

We initially discretize the domain into small cells of dimensions $2 \mathrm{~cm} \times 2 \mathrm{~cm} \times 2 \mathrm{~cm}$. The image obtained from TV minimization is then resized to cells of dimensions $4 \mathrm{~cm} \times 4 \mathrm{~cm} \times 4 \mathrm{~cm}$ in order to reduce the computation time of the loopy belief propagation algorithm. The intensity values of the image obtained from TV are normalized to lie in the range from 0 to 1 . Furthermore, those values in the top $1 \%$ and bottom $1 \%$ are directly mapped to 1 and 0 respectively, since they are inferred so close to $1 / 0$, with a very high confidence. The stopping criteria for the belief propagation algorithm is $10^{-4}$ for the mean change in beliefs, with a maximum of 100 iterations. The information about the outer boundary of the area may be known using cameras or laser scanners. However, only the cells on the boundary (i.e., the last layer of cells on the outer edge) would be known to be occupied by a wall in such a case, and the rest of the outer walls need to be imaged, as we shall show next. We next discuss the imaging results for the two scenarios.

\section{D Imaging Results}

Here, we show the experimental 3D imaging results for the two areas shown in Fig. 9. Fig. 10 (left) shows the region of interest for the two-cube scenario and Fig. 10 (middle) shows the 3D binary ground-truth image of the area. Fig. 10 (right) then shows the 3D reconstructed image from our proposed approach, using only $3.84 \%$ measurements. The percentage measurements refers to the ratio of the total number of measurements to the total number of unknowns in the discretized space (corresponding to the cells of dimensions $4 \mathrm{~cm} \times 4 \mathrm{~cm} \times 4 \mathrm{~cm}$ ), expressed as a percentage. As can be seen, the inner structure and the outer walls are imaged well, and the variations in the structure along the $\mathrm{z}$ direction are clearly visible. For instance, as the figure shows, the distance to the wall from the center of the top part is imaged at $1.50 \mathrm{~m}$, which is very close to the real value of $1.48 \mathrm{~m}$.

We next consider imaging the L-shape area. Note that we are imaging a larger area as compared to the two-cube scenario in this case. Fig. 11 (left) shows the region of interest for the L-shape area while Fig. 11 (middle) shows the 3D binary ground-truth image of the area. Fig. 11 (right) then shows the 3D image obtained from our proposed approach using only $3.6 \%$ measurements. As can be seen, the area is imaged well and the L shape of the structure is observable in the reconstruction. Furthermore, the distance to the wall from the center of the top part is imaged at $1.12 \mathrm{~m}$, which is very close to the real value of $1.08 \mathrm{~m}$. It is noteworthy that the 
Area of Interest - Top View

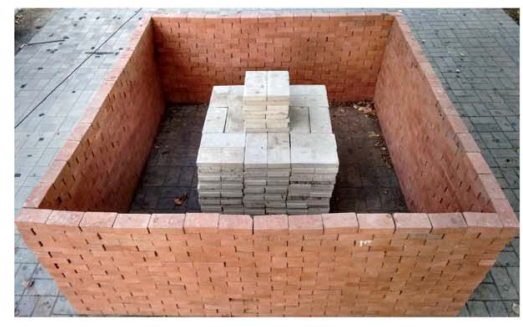

3D binary ground-truth image of the unknown area to be imaged $(2.96 \mathrm{~m} \times 2.96 \mathrm{~m} \times 0.4 \mathrm{~m})$

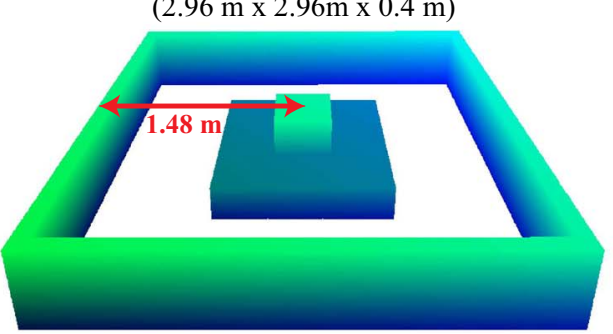

Our 3D image of the area, based on $3.84 \%$ measurements

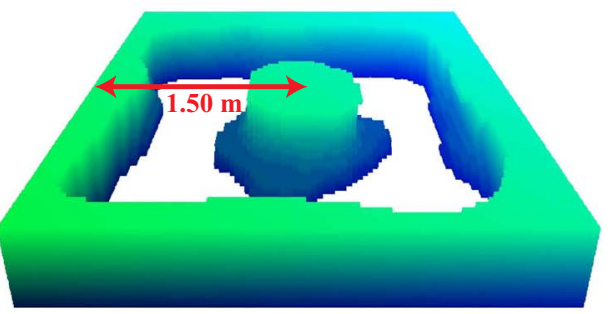

Figure 10: (left) The area of interest for the two-cube scenario, (middle) 3D binary ground-truth image of the unknown area to be imaged, which has the dimensions of $2.96 \mathbf{m} \times 2.96 \mathbf{m} \times 0.4 \mathbf{~ m}$, and (right) the reconstructed 3D binary image using our proposed framework.

Area of Interest - Top View

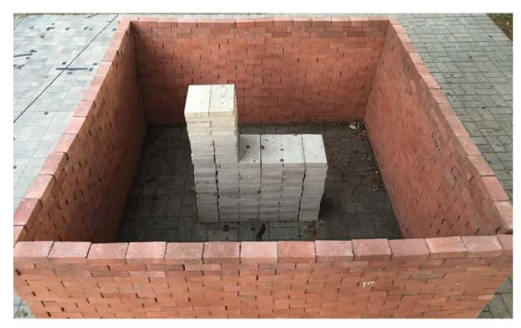

3D binary ground-truth image of the unknown area to be imaged $(2.96 \mathrm{~m} \times 2.96 \mathrm{~m} \times 0.5 \mathrm{~m})$

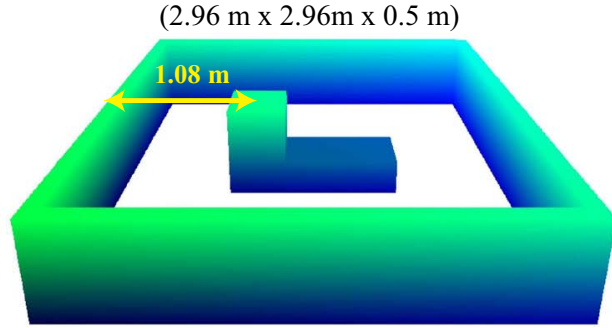

Our 3D image of the area, based on $3.6 \%$ measurements

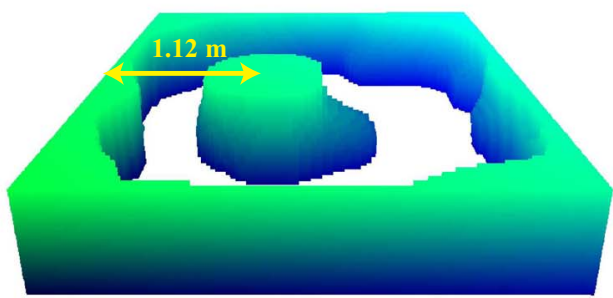

Figure 11: (left) The area of interest for the L-shape scenario, (middle) 3D binary ground-truth image of the unknown area to be imaged, which has the dimensions of $2.96 \mathbf{m} \times 2.96 \mathbf{m} \times 0.5 \mathbf{m}$, and (right) the reconstructed 3D binary image using our proposed framework.

inner two-cube structure is imaged at the center, while the inner L-shape structure is imaged towards the left, capturing the true trends of the original structures. Overall, the results confirm that our proposed framework can achieve 3D through-wall imaging with a good accuracy.

We next show a few sample 2D cross sections of the binary $3 \mathrm{D}$ images of Fig. 10 and 11. Fig. 12 (a) and (d) show two horizontal cross sections of the 3D binary ground-truth image of the two-cube area of Fig. 9 (a), while Fig. 12 (b) and (e) show the corresponding cross-sections in our reconstructed 3D image. Similarly, Fig. 13 (a) and (d) show two horizontal cross sections for the L-shape area of Fig. 9 (b), while Fig. 13 (b) and (e) show the corresponding images reconstructed from our proposed framework. In both cases, the different shapes and sizes of the inner structures at the two imaged cross sections are clearly observable.

\section{Comparison with the State-of-the-art}

In this section, we compare the proposed 3D imaging approach with the state-of-the-art for through-wall imaging with WiFi RSSI. More specifically, in the current literature [12,31], robotic through-wall imaging with WiFi power measurements is shown in 2D, with an approach that comprises of the measurement model described in Section 2, and sparse signal processing based on Total Variation minimization. However, directly extending the $2 \mathrm{D}$ approach for $3 \mathrm{D}$ imaging results in a poor performance. This is due to the fact that
3D imaging is a considerably more challenging problem, due to the severely under-determined nature of the linear model described in Section 2. Furthermore, by utilizing four measurement routes in the $2 \mathrm{D}$ case, every cell in the unknown domain (i.e., a plane in the case of 2D) appears multiple times in the linear system formulation. However, in the case of 3D imaging, there are many cells in the unknown domain that do not lie along the line connecting the TX and RX for any of the measurement routes, thereby never appearing in the linear system formulation. Thus, there is a higher degree of ambiguity about the unknown area in $3 \mathrm{D}$, as compared to the $2 \mathrm{D}$ counterpart, which could have only been avoided by collecting a prohibitive number of measurements. Therefore, the contributions of this paper along the lines of MRF modeling, loopy belief propagation, and 3D efficient path planning are crucial to enable 3D imaging.

In order to see the performance when directly extending the prior approach to 3D, we next compare the two approaches for the imaging scenarios considered in the paper. Consider the two-cube area of Fig. 9 (a). Fig. 12 (c) and (f) show the corresponding 2D cross sections of the $3 \mathrm{D}$ image obtained by utilizing the prior imaging approach [31] for our 3D problem. Similarly, for the L-shape area of Fig. 9 (b), Fig. 13 (c) and (f) show the corresponding 2D cross sections of the $3 \mathrm{D}$ image obtained by utilizing the prior imaging approach [31] for our 3D problem. 
Ground-truth image

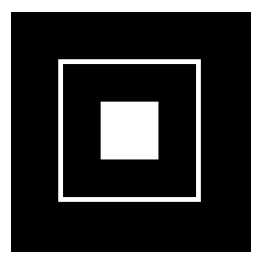

(a)

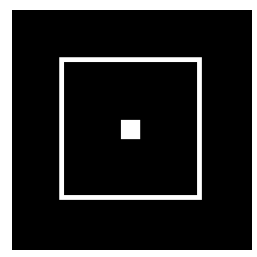

(d)
Proposed 3D imaging Prior 2D imaging approach approach

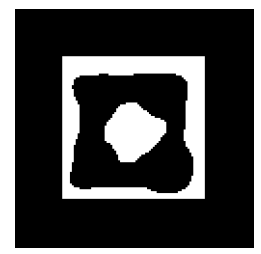

(b)

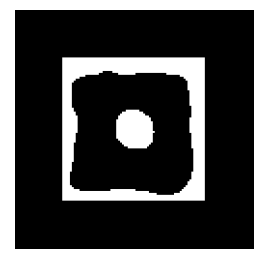

(e) directly extended to $3 \mathrm{D}$

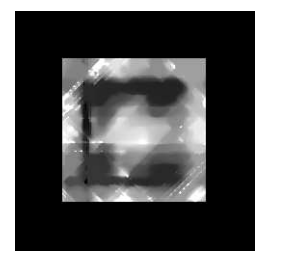

(c)

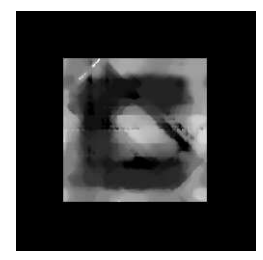

(f)
Ground-truth image

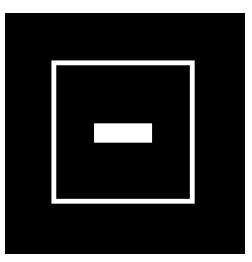

(a)

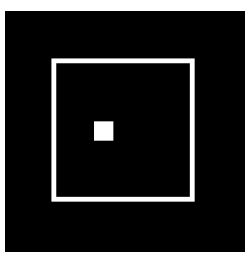

(d)
Proposed 3D imaging Prior 2D imaging approach approach

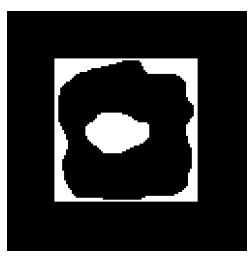

(b)

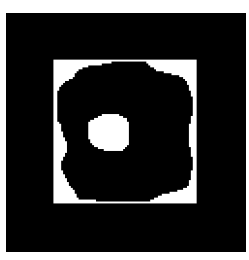

(e) directly extended to 3D

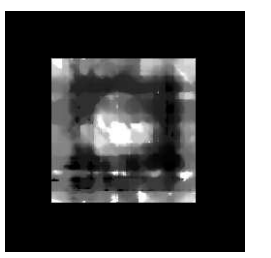

(c)

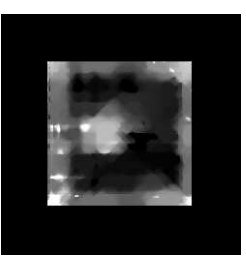

(f)
Figure 12: Sample 2D cross-sections of the 3D imaging results for the two-cube scenario. (a) and (d) show two $2 D$ cross sections of the ground-truth image, (b) and (e) show the corresponding cross sections of the imaging results obtained from the 3D imaging approach proposed in this paper, and (c) and (f) show the corresponding $2 \mathrm{D}$ cross sections of the 3D image obtained by directly extending the state-of-the-art imaging approach [31] to 3D.

As can be seen, it is challenging to obtain a good 3D reconstruction when directly utilizing the prior approach that was successful for imaging in 2D. There exists significant noise in the image due to the under-determined nature of the system and modeling errors. On the other hand, by incorporating Markov Random Field modeling and solving for the occupancy of each cell via utilizing loopy belief propagation, as we have done in this paper, we can see that the shapes and locations of the objects are reconstructed considerably more clearly.

\section{POSSIBLE FUTURE EXTENSIONS}

In this paper, we assumed that the unmanned vehicles can move on all sides of the area of interest. As part of the future work, considering the scenario where the UAVs can only access one side of the area of interest would be of interest. In this case, a new method for the optimization of the TX/RX positions is needed that restricts the positions to only one side of the area. Furthermore, environmental factors like extreme winds and minimal lighting can affect the performance of the Google Tangos and as a result the positioning performance of the UAVs, which will impact the overall imaging performance. A more advanced localization or joint imaging and localization can then possibly address these issues as part of future work.

\section{CONCLUSIONS}

In this paper, we have considered the problem of 3D through-wall imaging with UAVs, using only WiFi RSSI measurements, and proposed a new framework for reconstructing the 3D image of an unknown area. We have utilized an LOS-based measurement model
Figure 13: Sample 2D cross-sections of the 3D imaging results for the L-shape scenario. (a) and (d) show two $2 D$ cross sections of the ground-truth image, (b) and (e) show the corresponding cross sections of the imaging results obtained from the 3D imaging approach proposed in this paper, and (c) and (f) show the corresponding $2 \mathrm{D}$ cross sections of the 3D image obtained by directly extending the state-of-the-art imaging approach [31] to 3D.

for the received signal power, and proposed an approach based on sparse signal processing, loopy belief propagation, and markov random field modeling for solving the 3D imaging problem. Furthermore, we have shown an efficient aerial route design approach for wireless measurement collection with UAVs. We then described our developed experimental testbed for 3D imaging with UAVs and WiFi RSSI. Finally, we showed our experimental results for highquality 3D through-wall imaging of two unknown areas, based on only a small number of WiFi RSSI measurements (3.84\% and 3.6\%).

\section{ACKNOWLEDGMENTS}

The authors would like to thank the anonymous reviewers and the shepherd for their valuable comments and helpful suggestions. The authors would also like to thank Lucas Buckland and Harald Schäfer for helping with the experimental testbed, and Arjun Muralidharan for proof-reading the paper. This work is funded by NSF CCSS award \# 1611254.

\section{REFERENCES}

[1] 3DR. 2015. 3D Robotics. (2015). http://www.3dr.com Online

[2] F. Adib, C. Hsu, H. Mao, D. Katabi, and F. Durand. 2015. Capturing the human figure through a wall. ACM Transactions on Graphics 34, 6 (2015), 219.

[3] F. Ahmad, Y. Zhang, and M.G. Amin. 2008. Three-dimensional wideband beamforming for imaging through a single wall. IEEE Geoscience and Remote Sensing Letters 5, 2 (2008), 176-179.

[4] A. Beeri and R. Daisy. 2006. High-resolution through-wall imaging. In Defense and Security Symposium. International Society for Optics and Photonics, 62010J$62010 \mathrm{~J}$.

[5] J. Besag. 1974. Spatial interaction and the statistical analysis of lattice systems. fournal of the Royal Statistical Society. Series B (Methodological) (1974), 192-236.

[6] A. Blake, P. Kohli, and C. Rother. 2011. Markov random fields for vision and image processing. Mit Press. 
[7] E. Candès, J. Romberg, and T. Tao. 2006. Robust uncertainty principles: Exact signal reconstruction from highly incomplete frequency information. IEEE Transactions on information theory 52, 2 (2006), 489-509.

[8] R. Chandra, A.N. Gaikwad, D. Singh, and M.J. Nigam. 2008. An approach to remove the clutter and detect the target for ultra-wideband through-wall imaging. fournal of Geophysics and Engineering 5, 4 (2008), 412.

[9] W.C. Chew. 1995. Waves and fields in inhomogeneous media. Vol. 522. IEEE press New York.

[10] W.C. Chew and Y. Wang. 1990. Reconstruction of two-dimensional permittivity distribution using the distorted Born iterative method. IEEE Transactions on Medical Imaging 9, 2 (1990), 218-225.

[11] M. Dehmollaian and K. Sarabandi. 2008. Refocusing through building walls using synthetic aperture radar. IEEE Transactions on Geoscience and Remote Sensing 46, 6 (2008), 1589-1599.

[12] S. Depatla, L. Buckland, and Y. Mostofi. 2015. X-ray vision with only WiFi power measurements using rytov wave models. IEEE Transactions on Vehicular Technology 64, 4 (2015), 1376-1387.

[13] S. Depatla, A. Muralidharan, and Y. Mostofi. 2015. Occupancy estimation using only WiFi power measurements. IEEE fournal on Selected Areas in Communications 33, 7 (2015), 1381-1393.

[14] A.J. Devaney. 1982. Inversion formula for inverse scattering within the Born approximation. Optics Letters 7, 3 (1982), 111-112.

[15] D.L. Donoho. 2006. Compressed sensing. IEEE Transactions on information theory 52, 4 (2006), 1289-1306.

[16] P.F. Felzenszwalb and D.P. Huttenlocher. 2006. Efficient belief propagation for early vision. International journal of computer vision 70, 1 (2006), 41-54.

[17] Chen Feng, Wain Sy Anthea Au, Shahrokh Valaee, and Zhenhui Tan. 2012 Received-signal-strength-based indoor positioning using compressive sensing. IEEE Transactions on Mobile Computing 11, 12 (2012), 1983-1993.

[18] A. Gonzales-Ruiz, A. Ghaffarkhah, and Y. Mostofi. 2014. An Integrated Framework for Obstacle Mapping with See-Through Capabilities using Laser and Wireless Channel Measurements. IEEE Sensors fournal 14, 1 (January 2014), $25-38$.

[19] A. Gonzalez-Ruiz and Y. Mostofi. 2013. Cooperative robotic structure mapping using wireless measurements - a comparison of random and coordinated sampling patterns. IEEE Sensors fournal 13, 7 (2013), 2571-2580.

[20] Google. 2015. Google Project Tango. (2015). https://get.google.com/tango/ Online.

[21] Google. 2015. Tango Android Application Repo. (2015). https://github.com/ googlesamples/tango-examples-c Online.

[22] K. Held, E.R. Kops, B.J. Krause, W.M. Wells, R. Kikinis, and H. Muller-Gartner. 1997. Markov random field segmentation of brain MR images. IEEE Transactions on Medical Imaging 16, 6 (1997), 878-886.

23] Q. Huang, L. Qu, B. Wu, and G. Fang. 2010. UWB through-wall imaging based on compressive sensing. IEEE Transactions on Geoscience and Remote Sensing 48 3 (2010), 1408-1415.
[24] OLogic Inc. 2015. ROSTango Repository. (2015). https://github.com/ologic/ Tango/tree/master/ROSTango/src/rostango Online.

[25] W.C. Jakes and D.C. Cox. 1994. Microwave mobile communications. Wiley-IEEE Press.

[26] T.L. Jensen, J.H. Jørgensen, P.C. Hansen, and S.H. Jensen. 2012. Implementation of an optimal first-order method for strongly convex total variation regularization. BIT Numerical Mathematics 52, 2 (2012), 329-356.

27] P. Kohli and P.H. Torr. 2005. Efficiently solving dynamic markov random fields using graph cuts. In Tenth IEEE International Conference on Computer Vision, Vol. 2. IEEE, 922-929.

[28] D. Koller and N. Friedman. 2009. Probabilistic graphical models: principles and techniques. MIT press.

[29] Q.H. Liu, Z.Q. Zhang, T.T. Wang, J.A. Bryan, G.A. Ybarra, L.W. Nolte, and W.T. Joines. 2002. Active microwave imaging. I. 2-D forward and inverse scattering methods. IEEE Transactions on Microwave Theory and Techniques 50, 1 (2002), 123-133.

[30] G. Loianno, G. Cross, C. Qu, Y. Mulgaonkar, J.A. Hesch, and V. Kumar. 2015. Flying smartphones: Automated flight enabled by consumer electronics. IEEE Robotics \& Automation Magazine 22, 2 (2015), 24-32.

[31] Y. Mostofi. 2013. Cooperative Wireless-Based Obstacle/Object Mapping and See-Through Capabilities in Robotic Networks. IEEE Transactions on Mobile Computing 12, 5 (2013), 817-829.

[32] Y. Mostofi, A. Gonzalez-Ruiz, A. Gaffarkhah, and D. Li. 2009. Characterization and modeling of wireless channels for networked robotic and control systems-a comprehensive overview. In 2009 IEEE/RSf International Conference on Intelligen Robots and Systems. IEEE.

[33] Q. Pu, S. Gupta, S. Gollakota, and S. Patel. 2013. Whole-home gesture recognition using wireless signals. In Proceedings of the 19th annual international conference on Mobile computing \& networking. ACM, 27-38.

[34] Y. Rachlin, J.M. Dolan, and P. Khosla. 2005. Efficient mapping through exploitation of spatial dependencies. In Intelligent Robots and Systems, 2005.(IROS 2005). 2005 IEEE/RSf International Conference on. IEEE, 3117-3122.

[35] S.E. Shimony. 1994. Finding MAPs for belief networks is NP-hard. Artificial Intelligence 68, 2 (1994), 399-410.

[36] Y. Wang and A.E. Fathy. 2010. Three-dimensional through wall imaging using an UWB SAR. In 2010 IEEE Antennas and Propagation Society International Symposium. IEEE, 1-4.

[37] Y. Weiss. 1997. Belief propagation and revision in networks with loops. (1997).

[38] J. Wilson and N. Patwari. 2010. Radio tomographic imaging with wireless networks. IEEE Transactions on Mobile Computing 9, 5 (2010), 621-632.

[39] J.S. Yedidia, W.T. Freeman, Y. Weiss, and others. 2000. Generalized belief propagation. In NIPS, Vol. 13. 689-695.

[40] Z. Yin and R. Collins. 2007. Belief propagation in a 3D spatio-temporal MRF for moving object detection. In 2007 IEEE Conference on Computer Vision and Pattern Recognition. IEEE, 1-8.

[41] W. Zhang, A. Hoorfar, and L. Li. 2010. Through-the-wall target localization with time reversal music method. Progress In Electromagnetics Research 106 (2010), 75-89. 